\title{
CONTRATO DE TRABAJO, COMPROMISO Y SATISFACCIÓN: MODERACIÓN DE LA EMPLEABILIDAD
}

\author{
WORK CONTRACTS, COMMITMENT AND JOB SATISFACTION: MODERATED BY \\ EMPLOYABILITY \\ CONTRATO DE TRABALHO, COMPROMISSO E SATISFAÇÃO: MODERAÇÃO PELA EMPREGABILIDADE
}

\section{RESUMEN}

La búsqueda de prácticas flexibles de gestión de los recursos humanos viene desempeñando un papel importante para el desarrollo estratégico de las organizaciones y en particular en contextos de profunda crisis económica y financiera como la actual en Portugal, con una situación de desempleo creciente y un clima generalizado de inseguridad laboral. Este estudio pretende examinar si la percepción de empleabilidad por parte de los trabajadores puede desempeñar un papel moderador en la relación entre formas más flexibles de contratación, como el trabajo temporal, y las actitudes laborales. Resultados sugieren que la percepción de empleabilidad puede tener un efecto moderador en la relación entre el tipo de contrato de trabajo y el compromiso afectivo y la satisfacción laboral de los trabajadores bajo contrato directo con la organización pero esta interacción no se ha encontrado para trabajadores con contrato de trabajo temporal con las Agencias de Empleo.

PALABRAS CLAVE Empleabilidad, compromiso, satisfacción laboral, trabajo temporal.

Francisco Santos Cesário fcesario@ispa.pt

Doctor en Ciencias del Trabajo, Instituto Superior de Psicologia Aplicada - Lisboa, Portugal

Carlos Guillén Gestoso carlos.guillen@uca.es

Profesor del Departamento de Psicología, Universidad de Cádiz - Cádiz, España

F. Manuel Montalbán Peregrín fmontalban@uma.es

Profesor del Facultad de Estudios Sociales y del Trabajo, Universidad de Málaga - Málaga, España

\begin{abstract}
The search for flexible practices of human resources management comes playing an important role for the strategic organizational development and specially in contexts of deep economic and financial crisis like the present one in Portugal, with an increasing unemployment and a generalized climate of job insecurity. This study tries to examine if perception of employability by employees moderates the relationship between flexible forms of hiring, such as temporary work, and job attitudes like affective commitment and job satisfaction. Results suggest the moderating role of employability for workers under direct contract with the organization but this interaction has not been pointed for workers with an temporary work contract with Employment Agencies.

keywords Employability, commitment, job satisfaction, temporary employment.

Resumo A busca por práticas flexíveis da gestão dos recursos humanos vem desempenhando um papel importante para o desenvolvimento estratégico das organizações e, em particular, nos contextos da profunda crise econômica e financeira como a que vive hoje Portugal, com uma situação de desemprego crescente e um clima generalizado de insegurança laboral. Este estudo pretende examinar se a percepção de empregabilidade por parte dos trabalhadores pode desempenhar um papel moderador na relação entre as formas mais flexiveis de contratação, como o trabalho temporário, e as atitudes laborais. Resultados sugerem que a percepção de empregabilidade pode ter um efeito moderador na relação entre o tipo de contrato de trabalho e o compromisso efetivo e a satisfação laboral dos trabalhadores com contrato direto com a organização, mas esta interação não foi encontrada para os trabalhadores com contrato de trabalho temporário com as Agências de Emprego.
\end{abstract}

Palavras-chave Empregabilidade, compromisso, satisfação laboral, trabalho temporário. 


\section{INTRODUCCIÓN}

Hemos visto que a lo largo del 2011 por toda Europa se han ido acentuando dramáticamente los impactos de la actual crisis económica y financiera en los mercados de empleo. Las organizaciones están inquietas y tratan de reaccionar a los efectos negativos de la contracción económica aplicando estrategias y programas de retención radical de costes. Los medios de comunicación anuncian casi diariamente reducciones drásticas de la cantidad de puestos de trabajo mientras tanto las decisiones de contratación o de creación de nuevos trabajos son aplazadas. El ingreso al mercado de trabajo está cada vez más obstaculizado. Y como esas medidas iniciales no son suficientes para alcanzar las metas de reducción definidas, en algunos países, como en Portugal, tanto en el sector público como en el sector privado se avanza, complementariamente, no sólo hacia la suspensión de las actualizaciones anuales de sueldos sino también hacia la propia reducción de los sueldos y congelamiento de bonos o premios por desempeño, instrumentos tradicionalmente importantes y utilizados por las Direcciones de Recursos Humanos en sus prácticas de gestión de la motivación de sus colaboradores.

La fuerte presión que recayó sobre las organizaciones para que se mantuvieran competitivas y sobrevivieran a la crisis hace imprescindible que las prácticas de gestión de los recursos humanos sean evaluadas y reajustadas como elementos dinamizadores de mejor desempeño organizacional. Una mayor flexibilidad laboral es una prioridad en la agenda de las preocupaciones estratégicas de las organizaciones que buscan en la aplicación de prácticas de contratación de mano de obra más variable, un medio prudente para responder a las necesidades organizacionales y minimizar los costes fijos del personal. Una de las formas para lograr esa flexibilidad laboral es recurrir a diferentes tipos de contratación, frecuentemente podemos encontrar trabajadores con contratos directos con la organización, permanentes pero también contratos a tiempo fijo, y trabajadores con contratos con agencias de trabajo temporal.

En este contexto impredecible y difícil, el papel de la función de los Recursos Humanos es un tema candente. Nunca ha sido tan pertinente como lo es hoy la demanda del establecimiento de una relación directa entre las prácticas de GRH y el desempeño organizacional. ¿Será que nos aproximamos a abrir la caja negra propuesta por Bowen y Ostroff (2004)? El creciente interés en la búsqueda de evidencias en este sentido ha sido tan entusiástico como angustiante para los investigadores ya desde el final de la década del 90, y en especial desde el 2008 con el surgimiento de esta profunda época de crisis no prevista, en la que los más optimistas afirman que no empezará a desaparecer antes del 2015. La relación entre las prácticas de GRH y Desempeño Organizacional, es una cuestión sin respuesta inmediata. Un mito, como si aún estuviésemos en busca del santo grial, el Holy Graal de Boselie, Dietz y Boon (2005) o en busca de un efecto catalizador de búsqueda de nuevos enfoques para la misión de la función Recursos Humanos en tiempo de crisis.

Pero mientras no encontramos las respuestas más adecuadas, es cierto que los objetivos organizacionales no se logran sin un fuerte compromiso afectivo y satisfacción por parte de los colaboradores en los resultados de la organización. También las lecciones recibidas de tiempos anteriores de incertidumbre laboral indican que un entorno de inseguridad de empleo es una variable que perjudica las actitudes de los trabajadores y, de esa forma, es importante que las organizaciones identifiquen modos de atenuar ese tipo de efectos. En este estudio, de la misma forma que en las referencias de otros autores (DE WITTE, 1999; SVERKE y NASWALL, 2002; CHENG y CHAN, 2008) se considera que la empleabilidad podrá tener ese efecto en la relación entre el tipo de contrato de trabajo, con niveles distintos de inseguridad laboral, y el compromiso afectivo y la satisfacción de estos trabajadores.

Surge así, la necesidad de que la GRH busque un nuevo enfoque para mantener niveles de desempeño y de motivación adecuados a los objetivos de la eficiencia organizacional: apostar en prácticas innovadoras que promuevan la empleabilidad de sus colaboradores.

\section{EL CONCEPTO DE EMPLEABILIDAD}

El concepto de empleabilidad ha sido, de un modo general, asociado con la capacidad, mayor o menor, que tenemos para encontrar un trabajo ya sea en la situación de búsqueda del primer empleo como cuando pretendemos encontrar otro trabajo alternativo en caso de necesidad (BERNTSON y otros, 2006; BERNTSON y MARKLUND, 2007; SILLA y otros, 2009), 
particularmente debido al deseo de cambio opcional o porque nos encontramos en una situación de desempleo involuntario.

Particularmente en contextos coyunturales de recesión económica, de retracción de los mercados de trabajo, con búsqueda de mayor flexibilización de las relaciones laborales por parte de las organizaciones, con tasas de desempleo tan elevadas que hace mucho tiempo no se verificaban $(12,6 \%$ en julio de 2011 en Portugal), el concepto de empleabilidad asume un significado relevante ya que se trata de un nuevo mecanismo que puede conducir a la reducción de la percepción de inseguridad laboral (FORRIER y SELLS, 2003) y contribuir, de esta forma, a mejorar el bienestar laboral de los trabajadores dándoles una actitud más positiva frente a las incertidumbres del mercado de trabajo.

Este concepto ha despertado recientemente el interés de varios investigadores del área de psicología del trabajo y del comportamiento organizacional que sugieren el enfoque de la empleabilidad relacionada con dos niveles: a nivel macro, depende de las dinámicas de los mercados y de sus situaciones de oferta o de búsqueda y a nivel de la percepción individual dependiendo del grado de empleabilidad directamente basado en las competencias profesionales adquiridas y experiencia profesional que tiene consecuencias en las actitudes en el trabajo, en la satisfacción, en la implicación y en el bienestar laboral (FUGATE y KINICKI, 2008).

Como se observa en las definiciones presentadas (Cuadro 1), algunas parecen centrarse en las actitudes del trabajador, las definiciones de Hillage y Pollar (1998), de Fugate et al (2004), de Van der Heijde y Van der Heijden (2006) y la de Rothwell y Arnold (2007) pero en este estudio se adoptó una perspectiva más cercana a la indicada por Forrier y Sels (2003) porque se ha considerado que la percepción de empleabilidad del trabajador puede representar valor tanto internamente en la organización como también externamente en el mercado de empleo.

La empleabilidad interna se refiere a la posibilidad de un trabajador de sentir que tiene oportunidades de carrera en su actual trabajo, que sus competencias profesionales facilitan la rotación o movilidad interna, que la aplicación de su potencial se administra de forma adecuada y siente que tiene valor para la organización donde desarrolla en ese momento su actividad. Esta percepción de elevada empleabilidad interna podrá generar mayores niveles de satisfacción y de implicación o de compromiso afectivo para la organización. Elevada empleabilidad interna significa para el trabajador mayor probabilidad de mantener su empleo actual (DE WITTE, 2005).

La dimensión empleabilidad externa se refiere a la percepción de su valor en el mercado de trabajo, y siendo elevada, con la coyuntura del mercado favorable, la probabilidad de encontrar empleo en otra organización que le dé mejores perspectivas de desarrollo profesional o niveles superiores de compensación y de beneficios, es mayor. Una elevada percepción de empleabilidad externa podrá generar en el trabajador actitudes de confianza profesional, niveles elevados de motivación ya que desarrolla su actividad en un ambiente de mayor seguridad laboral, y si desea abandonar el trabajo o frente una situación imprevista de quiebra de vínculo laboral con la organización, el trabajador siente que podrá con más facilidad encontrar un empleo alternativo.

La percepción de empleabilidad permitirá, así, que los trabajadores adquieran una estabilidad dinámica que en el contexto actual pueda contribuir a su seguridad laboral. La permanencia en el mercado de trabajo, no en la misma organización en particular, será la esencia de un nuevo mundo laboral donde la calidad de trabajo

\section{Cuadro 1 - Ejemplos de definiciones de empleabilidad}

\section{Hillage y Pollard (1998)}

Forrier y Sells (2003)

Fugate et al. (2004)

Van der Heijde y Van der Heijden (2006)

Rothwell y Arnold (2007)
Capacidad de auto movimiento dentro del mercado de trabajo para aplicar el potencial en puestos de trabajo duraderos.

Oportunidad de un individuo para encontrar interna y/o externamente un empleo.

Una forma de adaptación específica y activa que permite a los trabajadores realizar e identificar las oportunidades de carrera.

Capacidad sistemática de obtener o de crear trabajo a través de la optimización de sus competencias.

Habilidad para mantener el trabajo actual u obtener otro que se desee. 
podrá considerarse una consecuencia de la empleabilidad (FORRIER y SELLS, 2003).

\section{GESTIÓN DE RR.HH., EMPLEABILIDAD Y DESEMPEÑO ORGANIZACIONAL}

Algunos modelos explicativos han indicado la existencia de una relación directa entre la eficacia de las prácticas de gestión de recursos humanos (GRH) y las actitudes (Figura 1) pero simultáneamente admiten que muchas de las prácticas de GRH podrían no tener ese efecto directo (BOSELIE y otros, 2005). Según esta perspectiva, la estrategia y las prácticas de GRH se diseñan para responder a los objetivos estratégicos organizacionales.

Las prácticas de Gestión de RR.HH. se aplican con el objetivo de alcanzar determinados Outcomes (actitudes y comportamientos). Se espera que "mejores" actitudes y comportamientos contribuyan para desarrollar un mejor desempeño interno (ej: crecimiento de niveles de productividad o de calidad del servicio). Ciertas prácticas de GRH podrán tener efecto directo en el desempeño interno sin impacto en los outcomes (ej: acciones a nivel de formación o rediseños de los procesos para mejorar habilidades y competencias con un impacto positivo en los niveles de productividad pero sin provocar modificaciones en los comportamientos o actitudes).

En este estudio se va a considerar específicamente en el análisis de las prácticas de gestión de recursos humanos (Figura 1) la práctica de contratación porque como se ha referido en la introducción, las organizaciones utilizan distintas formas de contratos de trabajo de acuerdo con sus necesidades de negocio y con distintos niveles de flexibilidad.

La introducción del papel de la Empleabilidad en el estudio de las actitudes de los trabajadores surge así, en la última década, con la intención de comprender mejor los resultados no concluyentes a los cuales se llegaba sobre los impactos que tenían las prácticas de GRH en las actitudes de los trabajadores en contexto de elevada inseguridad laboral. En especial, en tiempos de crisis de los mercados de trabajo, alguna investigación sugiere que los trabajadores estando fuertemente motivados para mostrar su valor en la organización donde realizan la actividad pretenden revelar niveles

\section{Figura 1 - Relación de las prácticas de gestión de RR.H.H. con outcomes y desempeño}

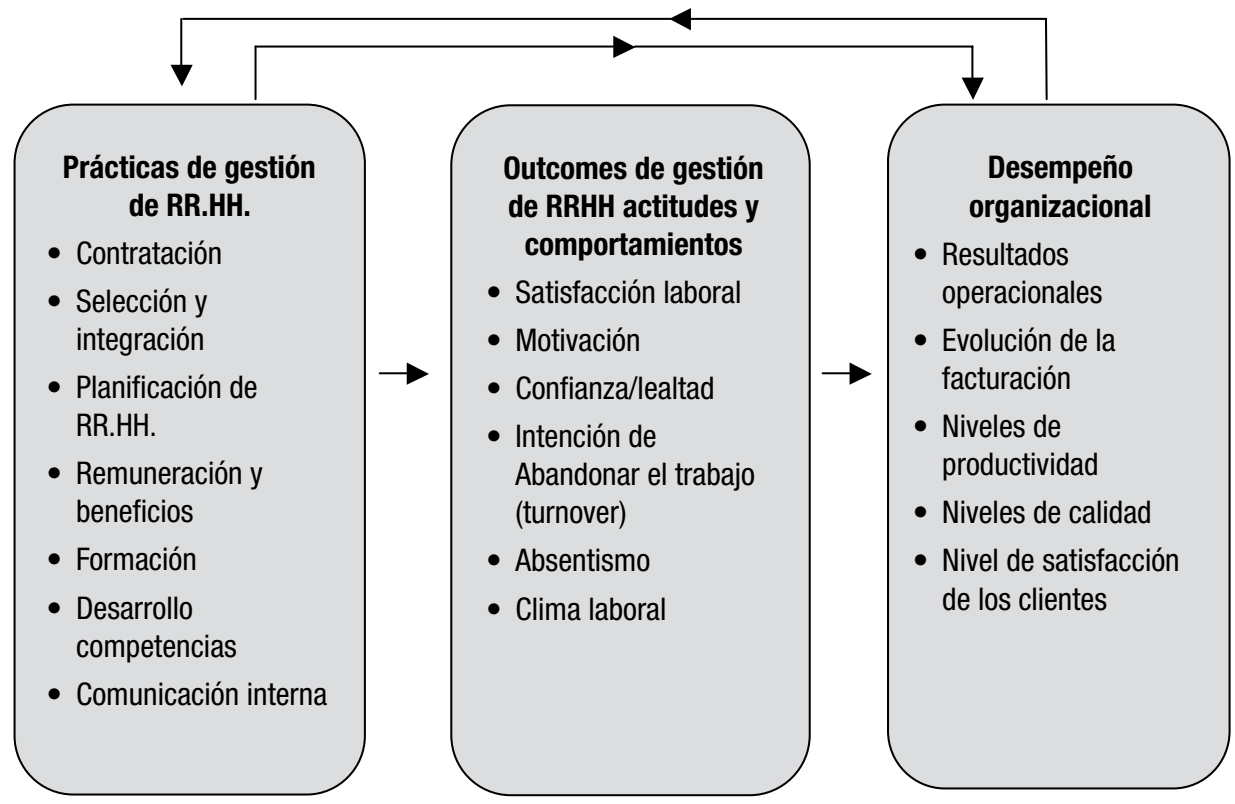

Fuente: Adaptado de Paauwee y Richardson (1997) 
elevados de compromiso como forma de aumentar la probabilidad de mantener sus puestos de trabajo en la organización (CONNELY y GALLAGHER, 2004). Su expectativa es alcanzar una elevada empleabilidad interna en la organización donde están y su percepción de elevada empleabilidad tiene un efecto positivo en su nivel de satisfacción y de compromiso afectivo para la organización.

En caso el contrato de trabajo termine, su expectativa, en situación de percepción de elevada empleabilidad, también es la de encontrar con mayor facilidad un empleo alternativo en otra organización que igualmente le proporcione niveles de satisfacción y de realización adecuados. Cuanto más se considera que la organización donde se ha trabajado promovió y apoyó el desarrollo de las competencias profesionales y que valorizó profesionalmente, mayor será la percepción de empleabilidad externa (HILLAGE y POLLARD, 1998).

Los resultados de estos estudios dan consistencia al valor que la empleabilidad asume para trabajadores tanto con contratos de trabajo permanentes como temporales y es posible que la percepción de empleabilidad represente un papel moderador (FORRIER y SELS, 2003, DE CUYPER y DE WITTE, 2005) entre las practicas de Gestión de RR.HH. y de las actitudes en el trabajo (Figura 2). Es probable que los trabajadores permanentes o temporales valoricen la empleabilidad como un nuevo mecanismo de protección frente a las variaciones constantes de los mercados de trabajo y que explican algunos de los impactos sobre sus actitudes en el trabajo (DE CUYPER y DE WITTE, 2007). El efecto de las prácticas de contratación sobre el compromiso organizacional o sobre la satisfacción laboral hacia los resultados organizacionales podrá ser reforzado cuando los empleados tengan una percepción de elevada empleabilidad.

Aunque la Gestión de RR.HH. no ha tenido obsesión por la medición, ya no hay excusas para no medir la percepción del grado de Empleabilidad de los trabajadores. Varios instrumentos están disponibles, unos con mayor número de ítems otros con menores pero más concisos y de mayor facilidad de aplicación, como es el caso de la escala Employment Outlook de Career Exploration Survey (STUMPF, COLARELLI y HARTMAN, 1983) que tiene solamente 3 ítems. Otra escala que es también muy utilizada y que tiene índices de calidad métrica comprobada fue desarrollada por DE WITTE (2000) con solamente 4 ítems y con 5 alternativas de respuesta tipo Liker que va desde 1 - Muy en desacuerdo hasta 5 - completamente de acuerdo. Los ítems que componen las dos escalas pueden observarse en el Cuadro 2.

La escala de Stumpf, Colarelli y Hartman (1983) contextualiza las respuestas sobre la percepción de empleabilidad en situación específica del mercado de trabajo, por eso ha sido adoptada para este estudio.

Así, el objetivo principal de este estudio es analizar si la empleabilidad desempeña un papel moderador en la relación entre diferentes prácticas de contratación utilizadas por una empresa y el compromiso y la satisfacción laboral de sus trabajadores. Para eso, se analiza también previamente si el tipo de contrato de trabajo

\section{Figura 2 - Empleabilidad: un posible efecto moderador}

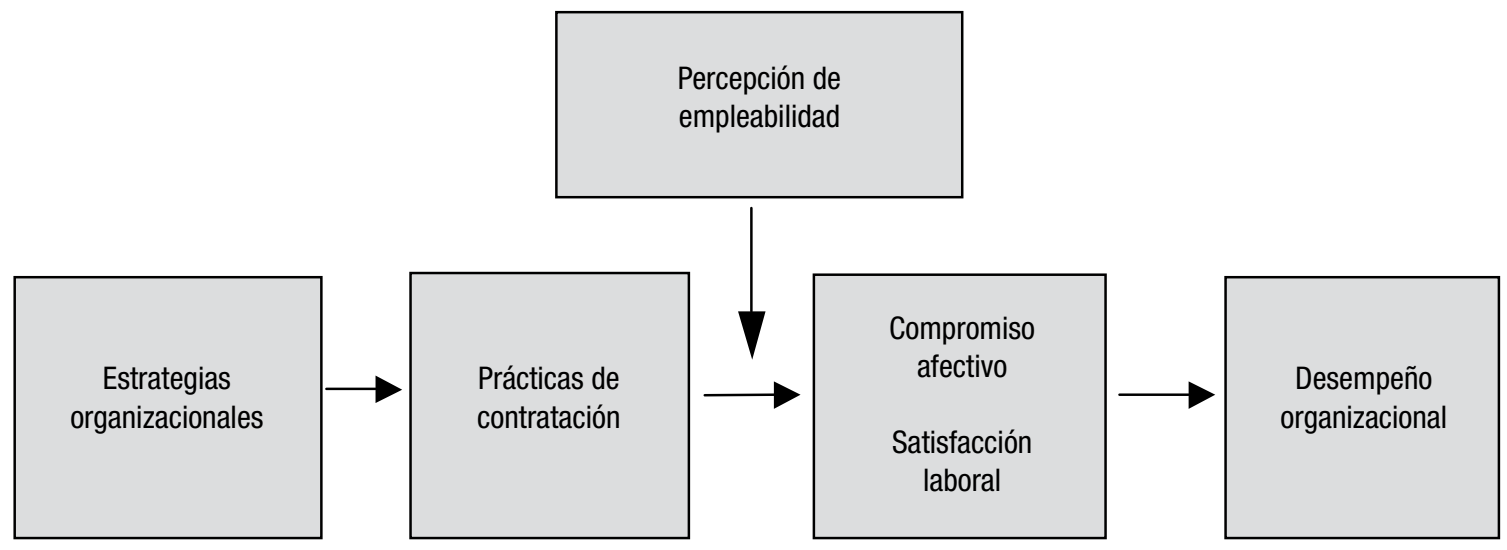


influye en la relación con esas actitudes, concretamente analizaremos si diferentes contratos de trabajo podrán afectar el compromiso afectivo y la satisfacción laboral. Como hemos descrito anteriormente, la empleabilidad, como mecanismo que el trabajador valora en una situación para mantener el empleo actual, o en situación de necesidad de encontrar otro empleo, podrá relacionarse con la incertidumbre asociada al tipo de contrato para explicar el compromiso afectivo y la satisfacción laboral de estos trabajadores. Las prácticas de contratación de recursos se analizarán por diferentes tipos de contrato de trabajo (término determinado y en régimen temporal a través de Agencias de Trabajo Temporal) y estableciendo como grupo de comparación la contratación sin término determinado (o indefinido). La decisión de realizar el estudio en una única organización se relaciona con el hecho de que sólo los trabajadores con diferentes contratos de trabajo, en funciones similares e influenciados por las mismas prácticas de gestión de recursos humanos, en la misma organización es posible analizar la influencia del tipo de contrato (CHAMBEL y CASTANHEIRA, 2006).

Hipótesis 1: El tipo de contrato de trabajo presenta una relación con el compromiso afectivo de los trabajadores y con la satisfacción laboral.

Hipótesis 2: La perspectiva de empleabilidad tiene un efecto moderador en la relación entre contrato de trabajo, compromiso y satisfacción.

\section{MÉTODO}

El estudio se apoyó en datos obtenidos por un cuestionario presentado directamente en una organiza- ción seleccionada en Portugal, líder de su sector de actividad (Contact Centers), con sede en Lisboa y con locales de trabajo distribuidos por varias regiones del país. La empresa, debido a su dimensión, cuenta con un número significativo de trabajadores a su servicio con diferentes tipos de contrato de trabajo. Después de obtenida autorización de la dirección de la empresa, el cuestionario se publicó en su intranet durante dos semanas, se envió un e-mail de divulgación del objetivo del estudio y de la importancia de la participación de los trabajadores con indicación de garantía de confidencialidad y anonimato de las respuestas. Responderán al cuestionario 1074 personas.

Los participantes son en su mayoría (64\%) una población joven con edad inferior a 30 años. Un 4,5\% presentan edad superior a 40 años. Aproximadamente el $44 \%$ son de género masculino y el $56 \%$ femenino. El sector de los Contact Center por la existencia de horarios de trabajo muy flexibles atrae un número elevado de estudiantes que así pueden conciliar su vida académica con una actividad remunerada (el 36\% de la muestra son estudiantes) e incluso después de terminar sus cursos permanecen en los Contact Center hasta encontrar una alternativa de trabajo más apropiada para sus calificaciones (el 30\% son licenciados). La distribución de la muestra por tipo de contrato permite ver el peso significativo de trabajadores con contrato de trabajo temporal a través de Agencias de Trabajo Temporal y cedidos a la empresa (89\%) y un número más reducido con contrato directamente con la empresa (11\%).

\section{Medidas}

Prácticas de contratación: las prácticas de contratación se midieron en cuatro tipos distintos de con-

\section{Cuadro 2 - Ejemplos de escalas de empleabilidad}

\section{Stumpf, Colarelli y Hartman (1983)}

En la situación actual del mercado de empleo es imposible encontrar un trabajo para el cual estoy preparado.

En la situación actual del mercado de empleo es posible ir a trabajar en una empresa de mi agrado.

En la situación actual del mercado de empleo mis cualidades y mi experiencia profesional me facilitan encontrar trabajo en otra empresa equivalente o mejor que la actual.

\section{De Witte (2000)}

Soy optimista, podré encontrar otro empleo si lo busco.

Sería fácil para mí encontrar otro empleo si perdiese este.

Si yo quisiera, iría con facilidad a trabajar para otra empresa.

Estoy seguro que podría encontrar rápidamente un empleo parecido a este. 
tratos de trabajo, lo que nos llevó a clasificarlos como categorías (tipo variable dummy), contrato con duración determinada directamente con la organización $(1=$ duración determinada, $0=$ otros $)$, temporal por la Agencia de Trabajo Temporal $(1=$ agencia temporal, $0=$ otros), contrato indefinido con la Agencia de Trabajo Temporal y cedido a la organización ( $1=$ indefinido Agencia, $0=$ otros $)$ y por fin el contrato de trabajo indefinido en la organización como grupo de comparación (AIKEN y WEST, 1991).

Actitudes de los trabajadores: el compromiso de los participantes con la empresa se midió con la escala de Allen y Meyer (1991) con los ítems de la dimensión afectiva (ej: Siento que formo parte de la familia de esta organización). La satisfacción general fue evaluada utilizando el cuestionario S10/12 de Melia y Peiró (1989) que constituyó la versión más reducida del Cuestionario de Satisfacción General en Organizaciones (1986), (ej: satisfacción para con la Supervisión o coordinación ejercida sobre sí).

Empleabilidad: los participantes respondieron a la percepción de su grado de empleabilidad en el mercado de trabajo a través de la escala CES (Career Exploration Survey) de Stumpf, Colarelli y Hartman (1983) (ítems descritos en el Cuadro2).

Variables de control: dado que los participantes son originarios de varios Contact Center distribuidos por el país y que la percepción de empleabilidad podrá estar relacionada con la calificación profesional y los mercados locales de empleo, se incluirán como variables de control las regiones (Norte, Centro, Sur y Lisboa) y el nivel de cualificación académica.

Las correlaciones encontradas entre las variables incluidas en este estudio, las consistencias internas, medias y desviaciones típicas se presentan en tabla 1.

\section{RESULTADOS}

Con el objetivode contrastar las hipótesis se realizó un análisis de regresión jerárquica modulado para verificar los efectos principales de cada tipo de contrato de trabajo sobre el compromiso afectivo y la satisfacción (H1) y también para observar el efecto de interacción de la variable moderadora empleabilidad (H2). Para analizar el efecto principal de diferentes tipos de contrato sobre las actitudes, se introdujeron en el primer paso de la regresión las variables de control educación y región de trabajo. El análisis de los datos tomó como categoría de comparación los trabajadores con contrato de duración indefinida con la organización y local de trabajo en la región de Lisboa.

La introducción de la variable de control nivel educación en el modelo añadía explicación para el compromiso y para la satisfacción con una relación negativa, cuanto mayor nivel de cualificación académica menor nivel de compromiso y de satisfacción. Se verifica una relación positiva entre región de trabajo y compromiso, empleados con local de trabajo en región Norte, Centro o Sur presentan un compromiso afectivo superior en comparación con los que trabajan en la región de Lisboa.

Los resultados muestran que la hipótesis de existencia de relación entre contrato de trabajo y compromiso afectivo y con la satisfacción se sostiene. En el segundo paso de la regresión (Tabla 2) cuando se introducen los diferentes tipos de contrato de trabajo se produce una mejora de la variancia explicada en el compromiso afectivo $\left(\Delta R^{2}=0,06, p<0,01\right)$ y en la satisfacción $\left(\Delta R^{2}=\right.$ $0,08, p<0,01)$. Se verificó una relación significativa en el compromiso afectivo para los trabajadores con un contrato de duración indefinida con la Agencia de Trabajo temporal $(\beta=-0,31, p<0,01)$, y también con la satisfac-

\section{Tabla 1 - Matriz de correlaciones y consistencia interna}

\begin{tabular}{|c|c|c|c|c|c|c|c|c|c|}
\hline & $\mathbf{M}$ & DP & 1 & 2 & 3 & 4 & 5 & 6 & $\alpha$ \\
\hline 1. Contrato duración determinada & & & 1 & & & & & & \\
\hline 2. Contrato indefinido con la ATT 1 & - & - & $0,095^{\star \star}$ & 1 & & & & & \\
\hline 3. Contrato temporal con la ATT 1 & - & $0,389^{\star *}$ & - & $0,710^{\star \star}$ & 1 & & & & \\
\hline 4. Empleabilidad & 0,016 & - & 0,030 & - & 0,029 & 1 & & & 0,75 \\
\hline 5. Satisfacción laboral & - & 0,027 & - & $0,271^{\star *}$ & $0,216^{\star \star}$ & $0,159^{\star \star}$ & 1 & & 0,93 \\
\hline 6. Compromiso afectivo & - & 0,013 & - & $0,230^{\star \star}$ & $0,129^{\star \star}$ & $0,221^{\star \star}$ & $0,703^{\star *}$ & 1 & 0,93 \\
\hline
\end{tabular}


ción $(\beta=-0,22, p<0,01)$, estos trabajadores muestran un nivel de compromiso y de satisfacción menor que los trabajadores con contrato de duración indefinida con la organización. No se verificó relación significativa para los empleados con contrato temporal con la Agencia o con contrato de duración determinada directamente con la organización. Estos resultados muestran que la hipótesis se confirma parcialmente y cuando se compara empleados con un vínculo indefinido directamente con la organización con los empleados con contrato indefinido con la Agencia de Trabajo Temporal.

La verificación de la segunda hipótesis, efecto moderador de la empleabilidad, se efectuó con la introducción de un tercer paso en el análisis de regresión y se probó el efecto de interacción de la empleabilidad y de cada tipo de contrato de trabajo sobre el compromiso afectivo y la satisfacción (Tabla 3).

Tal como se esperaba, en el tercer paso, se produce mejora de la variancia explicada del compromiso afectivo $\left(\Delta R^{2}=0,10, p<0,01\right)$. La interacción de la empleabilidad y contrato de trabajo resulta significativa y negativa para el compromiso de los empleados con contrato de duración determinada con la organización $(\beta=-0,11$, $\mathrm{p}<0,01)$ y con contrato temporal con la Agencia de Trabajo Temporal $(\beta=-0,39, p<0,01)$. Así se confirma el efecto moderador de la empleabilidad en la relación del contrato de trabajo y el compromiso afectivo. También se produce mejora de la variancia explicada de la satisfacción laboral en situación de percepción de empleabilidad ( $\left.\Delta \mathrm{R}^{2}=0,10, \mathrm{p}<0,05\right)$. La empleabilidad tiene un efecto moderador entre contrato de trabajo y satisfacción. La interacción de la empleabilidad y contrato resulta significativa para la satisfacción de los empleados con todo tipo de contrato de trabajo, indefinido con la Agencia $(\beta=-0,16, p<0,05)$, temporal con la Agencia $(\beta=-0,40, p<0,01)$ y de duración determinada directamente con la organización $(\beta=-0,11, \mathrm{p}<0,01)$.

Para dar más claridad a estos efectos de interacción y del papel de moderación de la empleabilidad se produjeron representaciones gráficas.

Las representaciones gráficas de los efectos de interacción (Figuras 3 y 4) muestran que los trabajadores cuando en percepción de situación de elevada empleabilidad presentan mayor nivel de compromiso afectivo. La variación más significativa de incremento del Compromiso se verifica para trabajadores con contrato permanente y con contrato de duración determinada con la organización (Figura 4) y más reducido para trabajadores con contrato temporal con la Agencia de Trabajo Temporal (Figura 3).

Los resultados muestran que los trabajadores con contratos de duración indefinida con la organización (permanentes) así como los trabajadores con contratos de duración indefinida con la Agencia de Trabajo Temporal (Figura 5) y los trabajadores con contrato de duración determinada con la organización (Figura 7), cuando en percepción de elevada empleabilidad en el mercado de trabajo, tienen mayor Satisfacción Laboral. Para estos trabajadores el hecho de que haya elevada empleabilidad en el mercado tiene impacto positivo en su nivel de satisfacción. Pero para trabajadores con contrato temporal con la Agencia no se verifica incre-

\section{Tabla 2 - Efectos principales del contrato de trabajo sobre el compromiso y la satisfacción}

\begin{tabular}{|c|c|c|}
\hline Pasos & $\begin{array}{c}\text { Compromiso afectivo } \\
\text { ( } \beta \text { último paso) }\end{array}$ & $\begin{array}{c}\text { Satisfacción } \\
\text { ( } \beta \text { último paso) }\end{array}$ \\
\hline $\begin{array}{ll}\text { Paso } 1 & \\
& \text { Educación } \\
& \text { Región Norte } \\
& \text { Región Centro } \\
& \text { Región Sur }\end{array}$ & $\begin{array}{l}-0,21^{\star \star \star} \\
0,17^{\star \star \star} \\
0,18^{\star \star \star} \\
0,26^{\star \star \star}\end{array}$ & $\begin{array}{l}-0,16^{\star \star \star} \\
0,07 \\
0,06 \\
0,22^{\star \star \star}\end{array}$ \\
\hline $\begin{array}{ll}\text { Paso } 2 & \\
& \text { Duración determinada } \\
& \text { Indefinido con la agencia } \\
& \text { Temporal con la agencia }\end{array}$ & $\begin{array}{l}-0,07 \\
-0,31^{\star \star *} \\
-0,09\end{array}$ & $\begin{array}{l}-0,01 \\
-0,22^{\star \star \star} \\
0,08\end{array}$ \\
\hline $\begin{array}{l}\Delta R^{2} \text { paso } 1 \\
\Delta R^{2} \text { paso } 2\end{array}$ & $\begin{array}{l}0,07^{\star \star \star} \\
0,06^{\star \star \star}\end{array}$ & $\begin{array}{l}0,04^{\star \star *} \\
0,08^{\star \star *}\end{array}$ \\
\hline
\end{tabular}

${ }^{* * *} p<0,01$ 
mento de su nivel de satisfacción laboral (Figura 6). La empleabilidad parece ser un factor atenuante del efecto del contrato de trabajo en la satisfacción laboral, cuando esta es elevada los trabajadores permanentes en la organización y con duración determinada muestran nivel de satisfacción muy similar (Figura 7).

Con este estudio ha sido posible verificar el efecto del tipo de contrato de trabajo en su relación con el com-

\section{Tabla 3 - Efectos de interacción de la empleabilidad sobre el compromiso y satisfacción}

\begin{tabular}{|c|c|c|}
\hline Pasos & $\begin{array}{l}\text { Compromiso afectivo } \\
\text { ( } \beta \text { último paso) }\end{array}$ & $\begin{array}{c}\text { Satisfacción } \\
\text { ( } \beta \text { último paso) }\end{array}$ \\
\hline $\begin{array}{l}\text { Paso } 1 \\
\text { Educación } \\
\text { Región Norte } \\
\text { Región Centro } \\
\text { Región Sur }\end{array}$ & $\begin{array}{r}-0,15^{\star \star \star} \\
0,16^{\star \star \star} \\
0,20^{\star \star \star} \\
0,26^{\star \star \star}\end{array}$ & $\begin{array}{l}-0,10^{\star \star \star} \\
0,06 \\
-0,07 \\
0,23^{\star \star \star}\end{array}$ \\
\hline $\begin{array}{l}\text { Paso } 2 \\
\text { Duración determinada } \\
\text { Indefinido con la agencia } \\
\text { Temporal con la agencia } \\
\text { Empleabilidad }\end{array}$ & $\begin{array}{l}-0,05 \\
-0,27^{\star \star \star} \\
-0,06 \\
0,62^{\star \star \star}\end{array}$ & $\begin{array}{l}0,01 \\
-0,18^{\star \star \star} \\
0,11 \\
0,59^{\star \star \star}\end{array}$ \\
\hline $\begin{array}{l}\text { Paso } 3 \\
\text { Indefinido agencia } x \text { empleabilidad } \\
\text { Temporal agencia } x \text { empleabilidad } \\
\text { Duración determinada } x \text { empleabilidad }\end{array}$ & $\begin{array}{l}-0,10 \\
-0,39^{\star \star} \\
-0,11^{\star \star}\end{array}$ & $\begin{array}{l}-0,16^{\star} \\
-0,40^{\star \star} \\
-0,11^{\star \star}\end{array}$ \\
\hline $\begin{array}{l}R^{2} \\
\Delta R^{2} \text { paso } 1 \\
\Delta R^{2} \text { paso } 2 \\
\Delta R^{2} \text { paso } 3\end{array}$ & $\begin{array}{l}0,07^{\star \star \star} \\
0,09^{\star \star \star} \\
0,01^{\star \star}\end{array}$ & $\begin{array}{l}0,04^{\star \star} \\
0,10^{\star \star \star} \\
0,01^{\star}\end{array}$ \\
\hline
\end{tabular}

${ }^{*} p<0,05 ;{ }^{* \star} p<0,10 ;{ }^{* \star \star} p<0,01$

Figura 3 - Interacción entre contrato y empleabilidad sobre el compromiso afectivo: empleados con contrato temporal con la agencia de trabajo temporal

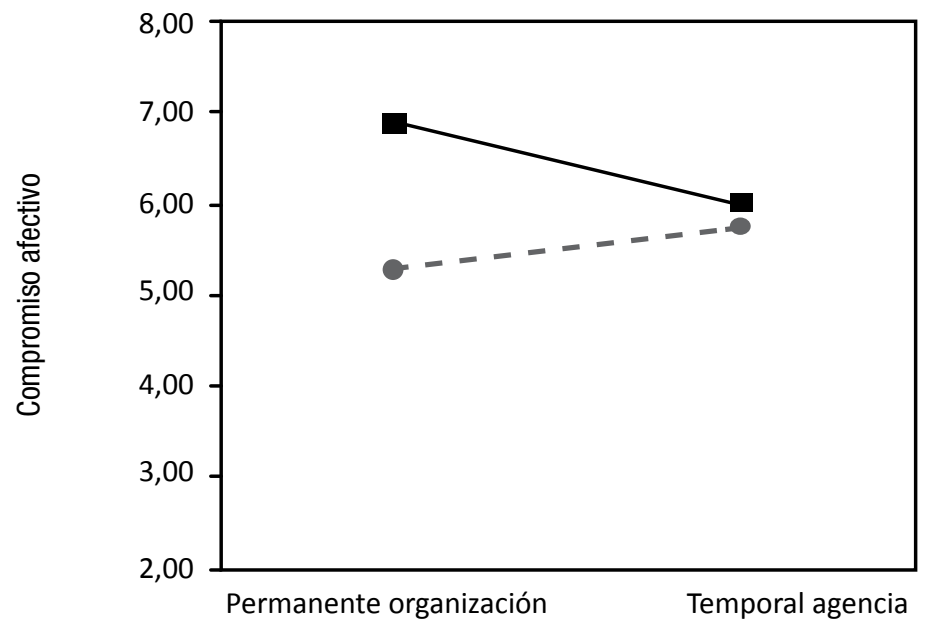

$\rightarrow$ Alta empleabilidad

- - Baja empleabilidad 
promiso afectivo y con la satisfacción laboral. Los trabajadores con contrato temporal con la Agencia exhiben en menor medida esas actitudes que los restantes. Incluso, tal como se esperaba, se verifica que la empleabilidad podrá atenuar el efecto del tipo de contrato en esas actitudes, observándose un efecto positivo atenuador tanto para trabajadores con contrato de trabajo temporal de Agencia como para los trabajadores con contrato de duración determinada directamente con la empresa.

En esta línea, de una forma general, el estudio confirmó la relación significativa entre tipo de contrato de trabajo y variables actitudes como el compromiso afectivo y la satisfacción laboral y bienestar de los empleados de acuerdo con investigación reciente en este tema (BERNHARD-OETTEL, SVERKE y DE WITTE, 2005; DE CUYPER y DE WITTE, 2006; BERNHARD-OETTEL, DE CUYPER, BERNTSON y ISAKSSON, 2008; GARCIA-MONTALVO, PEIRÓ y SORO, 2003; CASTANHEIRA y CHAMBEL, 2006,2007).

Tal como en estudios anteriores, los resultados

Figura 4 - Interacción entre contrato y empleabilidad sobre el compromiso afectivo: empleados con contrato de duración determinada con la organización

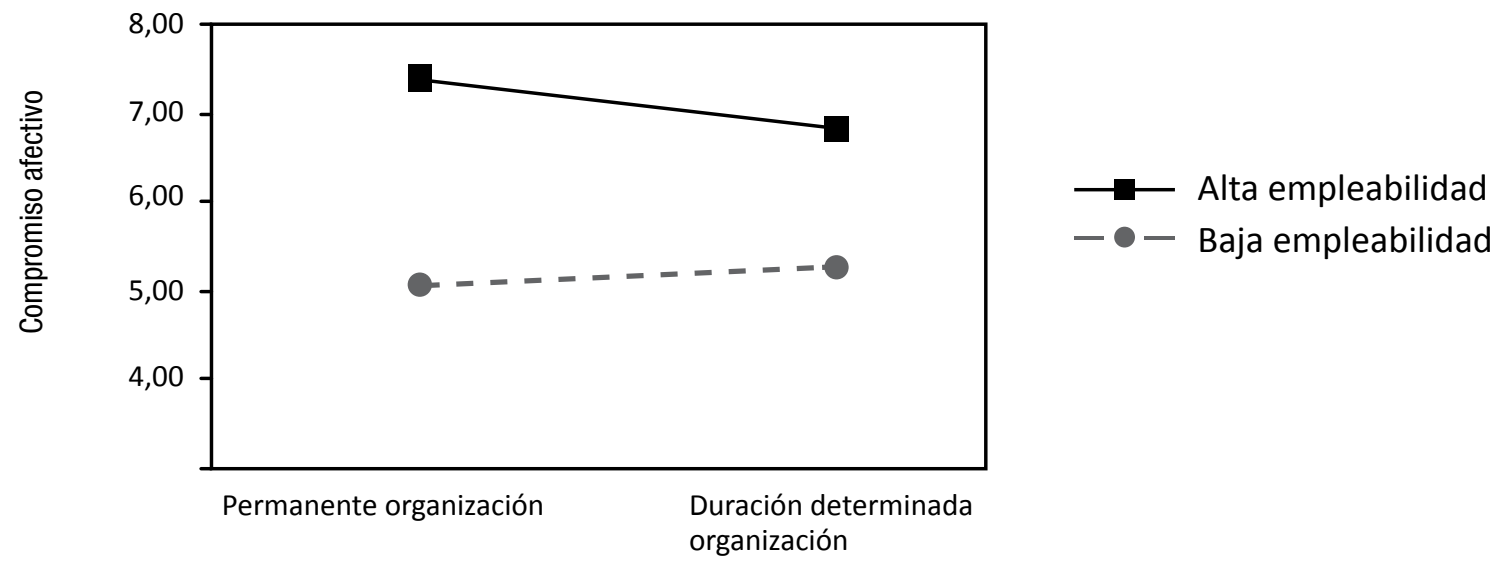

Figura 5 - Interacción entre contrato y empleabilidad sobre la satisfacción: empleados con contrato indefinido con la agencia
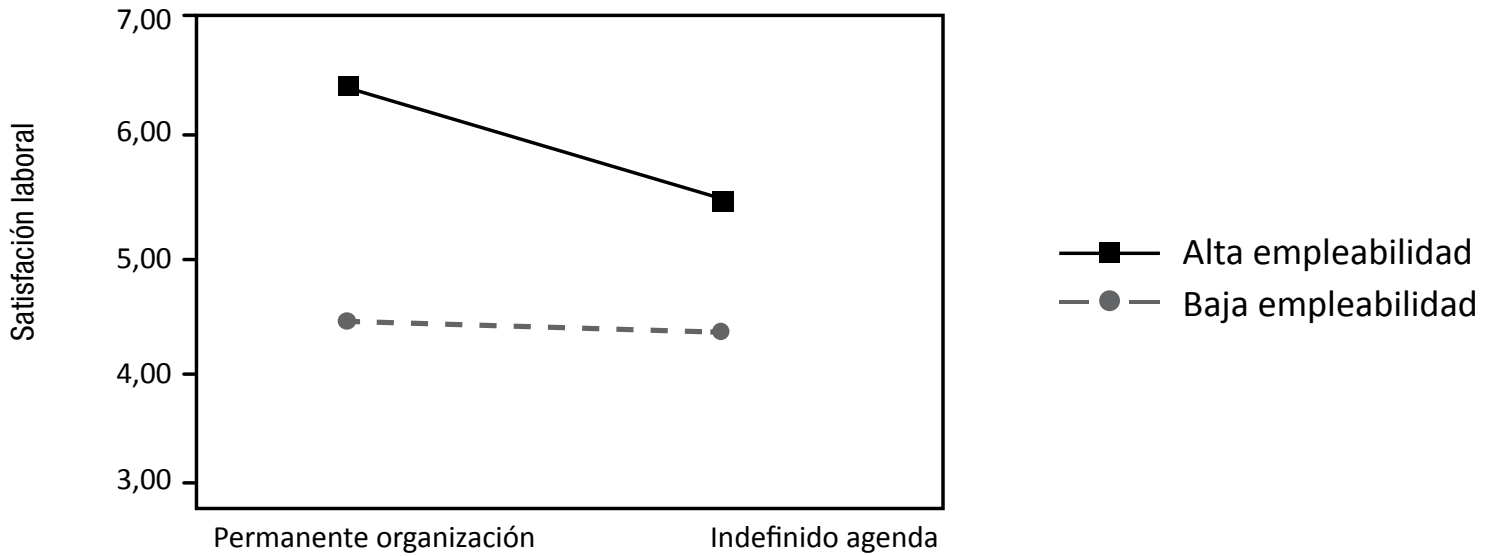
indican que el tipo de contrato de trabajo predice la satisfacción pero los estudios no son conclusivos. Concretamente en este estudio se han encontrado niveles más elevados de satisfacción en los empleados con contrato de trabajo permanente con la organización y los empleados con contrato temporal con la Agencia de Trabajo Temporal, en comparación con los niveles de los empleados con contrato permanente con la Agencia o con contrato de duración determinada directamente con la organización. A nivel de flexibilidad de contra- tación, son precisamente los trabajadores con contrato de trabajo permanente y los trabajadores con contrato temporal con la Agencia de Trabajo Temporal los que representan los extremos de la precariedad de empleo, la menor precariedad y la mayor precariedad.

Para los empleados con contrato permanente con la organización su percepción de elevada satisfacción puede resultar del hecho de sentir que trabajan en una organización que en su sector de actividad adopta las mejores prácticas de gestión de recursos humanos. El

\section{Figura 6 - Interacción entre contrato y empleabilidad sobre la satisfacción: empleados con contrato temporal con la agencia}
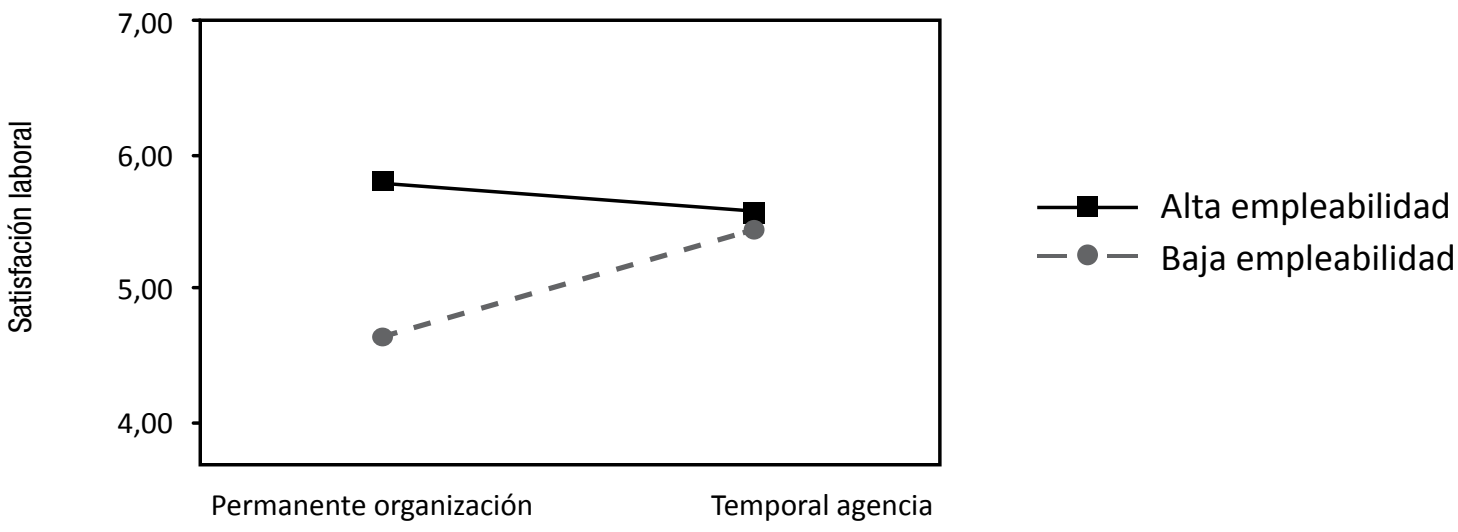

Figura 7 - Interacción entre contrato y empleabilidad sobre la satisfacción: empleados con contrato de duración determinada con la organización

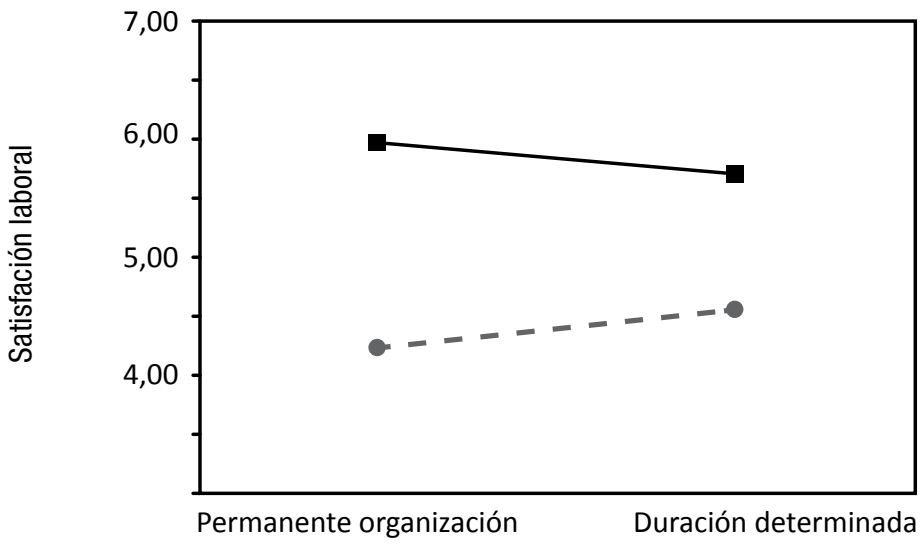

$\longrightarrow-$ Alta empleabilidad
- - Baja empleabilidad 
desarrollo de planes de carrera profesional ha sido importante, ya que la organización donde se realizó este estudio desarrolla anualmente programas de formación específicos para los recursos humanos de sus Contact Centers teniendo como objetivo mejorar sus competencias profesionales. La organización presenta una fuerte apuesta en las instalaciones y ambientes físicos de trabajo con espacios modernos. Son precisamente las dimensiones de satisfacción con los equipos de Supervisión y con los ambientes físicos, las dos dimensiones de la escala de Satisfacción Laboral utilizadas en este estudio.

Para los empleados en la organización con contratos temporales a través de la Agencia de Trabajo Temporal, su nivel elevado de satisfacción podrá estar influenciado por sentir que trabajan en una organización que no practica diferencias en la política de gestión de recursos humanos entre permanentes y temporales. La oportunidad de evolución profesional y de formación son las mismas, así como los sistemas de recompensa y remuneración. De este modo los temporales tienen una percepción de tratamiento justo y resulta en impacto en su nivel elevado de satisfacción.

Una perspectiva distinta podrá existir con los empleados que tienen contrato permanente con la Agencia de Trabajo Temporal. Cuando terminan su colaboración en esta organización, la Agencia los enviará a otra organización. Su nivel inferior de Satisfacción podrá resultar de la baja conexión con esta organización.

En investigaciones recientes conducidas en varios Países de la UE (Psycones Report) los resultados tampoco son conclusivos respecto a la relación entre mayor precariedad laboral y mayor nivel de insatisfacción (ISAKSSON, 2006). Los resultados no soportan la hipótesis de que trabajadores temporales presentarían nivel de insatisfacción laboral superior a los trabajadores con contratos permanentes y sus conclusiones generales no establecen una conexión fuerte entre tipo de contrato de trabajo y satisfacción laboral (BERNHARD-OETTEL, SVERKE y DE WITTE, 2005; DE WITTE y NASWALL, 2003; GUEST y CONWAY, 1997). Es importante destacar que los empleados temporales incluidos en este estudio no son temporales voluntarios. En Portugal no hay estadísticas que permitan distinguir trabajadores temporales voluntarios de los no voluntarios. Se parte del principio de que en este estudio todos los temporales lo son porque no han encontrado alternativa segura de empleo. Podría ser una línea futura de investigación constituir un estudio con un segundo grupo de temporales, las personas que buscan trabajo temporal porque sería su opción de vida y comparar su nivel de satisfacción laboral o de compromiso organizacional con los temporales no voluntarios. La realidad no permite hacer este estudio comparativo porque nuestra percepción es que el número de temporales por opción propia en el mercado de trabajo en Portugal es inexpresivo.

Los valores más elevados de compromiso afectivo encontrado se verifican con las personas con contrato de trabajo permanente con la organización. Este resultado, expresa un sentimiento de deseo de continuar en la organización. Pero se aprecia que inmediatamente surgen con elevados niveles de compromiso afectivo los trabajadores con contrato de trabajo temporal con la Agencia de Trabajo Temporal, lo que puede revelar una situación de elevada perspectiva de vínculo futuro a la organización y en consecuencia su nivel de compromiso no depende de la duración del contrato de trabajo.

Para estos empleados con contrato temporal a través de la Agencia de Trabajo Temporal, que prestan servicios en la organización y que mostraron elevado nivel de compromiso, podrá significar que sienten que colaboran con una organización que potencia los recursos internos independientemente del tipo de contrato de trabajo y que practica una política de selección para trabajo permanente entre sus colaboradores temporales y por eso pretenden mostrar a la organización que tienen valor y su elevado nivel de compromiso resulta de una expectativa fuerte de cambio de contrato de trabajo.

Como se esperaba, los trabajadores con menor nivel de compromiso son los empleados que tienen un contrato de duración indefinida con la Agencia de Trabajo Temporal y son por ésta colocados en actividad en empresas clientes de la Agencia. Estos son trabajadores que no tienen perspectiva de continuar en la organización y cuando terminen su prestación de servicio en esta organización serán colocados por la Agencia en otra organización, aunque pretendiesen continuar en ella.

El presente estudio presenta algunas limitaciones. La primera hace referencia a las medidas utilizadas para las variables dependientes Compromiso y Satisfacción y la variable de moderación Empleabilidad. Estas variables se han medido con escalas de auto-informe, de modo que los datos del cuestionario provienen del mismo participante que responde al mismo tiempo a todos los ítems de las tres escalas. El propio diseño transversal de la investigación condiciona la interpretación de las relaciones causales entre las variables y, de este modo, en investigaciones futuras se planteará con un diseño longitudinal. Esto se debe a que cuanto mayor 
se considere la duración de la experiencia profesional, se podrá obtener efectos positivos sobre la valorización del trabajador y en especial para el trabajador en contrato temporal. Por último, la escasez de literatura referente a investigaciones desarrolladas sobre muestras de trabajadores temporales en organizaciones en Portugal, limitado a estudios de Chambel (2006, 2007), sobre flexibilidad laboral y en particular sobre el papel de moderación probado para la Empleabilidad. Estas limitaciones de estudios en la realidad portuguesa no permitirán presentar comparación de resultados.

\section{CONCLUSIÓN}

El estudio de diferencias de actitudes entre trabajadores con contratos permanentes y temporales no son conclusivos, varios factores pueden contribuir para su explicación, desde la naturaleza de cada función, el tipo de tareas desarrolladas, los aspectos de clima y de cultura organizacional y las políticas y prácticas de gestión de los recursos humanos. El sistema adoptado en cada país para promover las cualificaciones profesionales, los mecanismos de protección social en situación de desempleo pueden también condicionar los resultados.

La recuperación de la economía podrá significar con la búsqueda de mayor flexibilidad laboral, un peso creciente de utilización de fuerza de trabajo en contratación temporal. Como los resultados mostraron, mayor precariedad laboral no significa menor satisfacción laboral y menor compromiso.

El empleo temporal puede representar para la población joven una puerta de entrada importante al mercado de trabajo y para los trabajadores en situación de desempleo no voluntario significará también una forma de aproximación a la actividad profesional y de actualización de cualificaciones. Los Gestores y Directivos de las organizaciones pueden observar las capacidades, los comportamientos y actitudes y los niveles de productividad de los trabajadores en situación temporal y pueden tomar decisiones fundamentadas en el desempeño y cambiar la situación contractual del empleado, de temporal a contrato permanente. Esta perspectiva, como un proceso de preselección, puede dar un mensaje muy positivo para la satisfacción y compromiso organizacional de los trabajadores temporales.

La introducción de la empleabilidad como variable de moderación constituye probablemente un aspecto de gran interés para investigaciones futuras. Es necesario mejorar la medición del grado de empleabilidad, definir factores que se constituyen como dinamizadores de la empleabilidad, conocer sistemáticamente las demandas del mercado, conocer lo que el mercado valoriza a nivel de cualificaciones. Este aspecto, deberá orientar las políticas nacionales y de la Unión Europea de promoción de la empleabilidad y del empleo. La flexibilización de las relaciones laborales deberá tener como objetivo un impulso hacia la creación de empleo cualificado. Para el sistema educativo, los contenidos de los currículos académicos deberán tomar en cuenta los aspectos orientados hacia el grado de elevada empleabilidad contribuyendo para reducir la inseguridad laboral de las nuevas generaciones.

En el contexto actual de elevados rasgos de competitividad para las organizaciones, con este trabajo ha sido posible verificar que se puede continuar contando con trabajadores con contratos de trabajo temporal a través de las Agencias, pero para estos trabajadores deben también aplicarse las prácticas de gestión de recursos humanos con el objetivo de desarrollar su nivel de empleabilidad. En realidad, contratar trabajadores de Agencia no significa que la organización no tenga también responsabilidad con estos trabajadores.

Las organizaciones desarrollan políticas de responsabilidad social externa muy difundidas en los medios de comunicación pero deben también desarrollar políticas de responsabilidad social interna, que significa promover el desarrollo de la empleabilidad de su fuerza de trabajo independientemente de su situación contractual, a través, por ejemplo, de formación cualificada, de rotación funcional, de enriquecimiento de las tareas y puestos de trabajo, de crear oportunidades de desarrollo profesional, etc. Cuanto más valor el trabajador tenga para la organización, más valorado estará en el mercado de trabajo. El paradigma obsoleto de promoción de empleo para la vida en la misma organización podrá ser sustituido así por la promoción de empleabilidad para la vida activa.

\section{REFERENCIAS}

ALLEN N. J; MEYER, J. P. A three-component conceptualization of organizational commitment. Human Resources Management Review, v. 1, n. 1, p. 61-89, 1991. 
BERNHARD-OETTEL, C; SVERKE, M; DE WITTE, H. Comparing three alternative types of employment with permanent full-time work: how do employment contract and perceived job conditions relate to health complains? Work E Stress, v. 19, n. 4, p. 301-318, 2005

BERNHARD-OETTEL, C; DE CUYPER, N; BERNTSON, E; ISAKSSON, K. Well-being and organizational attitudes in alternative employment: the role of contract and job preferences. International Journal of Stress Management, v. 15, n. 4, p. 345-363, 2008.

BERNTSON, E; MARKLUND, S. The relationship between perceived employability and subsequent health. Work and Stress, v. 2, n. 3, p. 279-292, 2007.

BERNTSON, E; SVERKE, M; MARKLUND, S. Predicting perceived employability: Human capital or labor market opportunities? Economic and Industrial Democracy, v. 27, n. 2, p. 223-244, 2006.

BOWEN, D; OSTROFF, C. Understanding HRM-Firm performance linkages: the role of the "strength" of the HRM system. Academy of Management Review, v. 29, n. 2, p. 203-221, 2004.

BOSElie, P; DieTZ, G; BOON, C. Commonalities and contradictions in HRM and performance research. Human Resource Management Journal, v. 15, n. 3, p. 67$94,2005$.

CHAMBEL M. J; CASTANHEIRA F. Different temporary work status, different behaviors in organization. Journal of Business and Psychology, v. 20, n. 3, p. 351-367, 2006.

CHAMBEL M. J; CASTANHEIRA F. They don't wont to be temporary workers: similarities between temps and core workers. Journal of Organizational Behavior, v. 28, n. 8 . p. 943-959, 2007.

CHENG, G; CHAN, D. Who suffers more from job insecurity? A meta-analytic review. Applied Psychology: An International Review, v. 57, n. 2, p. 272-303. 2008.

CIETT. The agency work industry around the world. EcOnomic report. Ciett National Federation. Brussels, 2011

CONNELY, C; GALLAGHER, D. Emerging trends in contingent work research. Journal of Management, v. 30, n. 6, p. 959-983, 2004.
DE CUYPER, N; DE WITTE H. Job insecurity: mediator or moderator in the relationship between type of contract and various outcomes? Journal of Industrial Psychology, v. 31, n. 4, p. 79-86, 2005.

DE CUYPER, N; DE WITTE, H. Job insecurity in temporary versus permanent workers: Associations with attitudes, well-being, and behaviour. Work and Stress, v. 21, n.1, p. 65-84, 2007.

DE CUYPER, N; DE WITTE, H. The management paradox: self-rated employability and organizational commitment and performance. Personnel Review, v. 40, n. 2, p. 152172, 2011.

DE CUYPER, N; DE WITTE, H; KINNUNEN, U; NATTI, J. The relationship between job insecurity and employability and well-being among Finnish temporary and permanent employees. International Studies of Management and Organization, v. 40, n. 1, p. 57-73, 2010.

DE WITTE, H. Job insecurity and psychological well-being: review of the literature and exploration of some unresolved issues. European Journal of Work and Organizational Psychology, v. 8, n. 2 p. 155-177. 1999.

DE WITTE, H. Work ethic and job insecurity: measurement and consequences for well-being, satisfaction and performance. In R. Bouwen, K, De Witte, H. De Witte y T. Taillieu ( Eds.) Van groep naar gemeenschap, p 325350. Leuven, 2000.

DE WITTE H. Job insecurity: review of the international literature on definitions, prevalence, antecedents and consequences. Journal of Industrial Psychology, v. 31, n. 4, p. 1-4, 2005.

DE WITTE, H; NASWALL, K. Objective versus subjective job insecurity: consequences of temporary work for job satisfaction and organizational commitment in four European countries. Economic and Industrial Democracy, v. 24, n 2, p. 149-188, 2003.

FORRIER, A; SELS, L. The concept employability: a complex mosaic. International Journal of Human Resources Development and Management, v. 3, n. 2, p. 102-124, 2003.

FRUTOS, B; RUIZ, M; SAN MARTÍN, R. Análisis factorial confirmatorio de las dimensiones del compromiso con la organización. Psicologia, v. 19, n. 3, p. 345-366, 1998. 
FUGATE, M; KINICKI, A. A dispositional approach to employability: development of a measure and test of implications for employee reactions to organizational change. Journal of Occupational and Organizational Psychology, 81, p. 503-527, 2008.

FUGATE, M; KINICKI, A; ASHFORTH. Employability: a psycho-social construct, its dimensions and applications. Journal of Vocational Behavior, v. 65, n. 1, p. 14-38, 2004.

GARCÍA-MONTALVO, J; PEIRÓ, J. M. y SORO, M. Capital Humano. Observatorio de Inserción Laboral de los Jóvenes:1996-2002. Bancaje-Ivie: Valência, 2003.

GUEST, D; CONWAY, N. Employee motivation and psychological contract. London. CIPD, 1997.

HILlAGE, J; POLARD, E. Employability: developing a framework for policies analysis. Research Brief 85, DFEE, 1998.

ISAKSSON, K..Psychological Contracts across Employment Situations (Psycones): Final Scientific Report, http://www. uv.es/ psycones, Acceso en 12.04.2006.

MELIÁ, J. L; PEIRÓ, J. M. El cuestionário de satisfacción S10/12: estrutura factorial, fiabilidad y validez. Revista de Psicologia del Trabajo y de las organizaciones, v. 4, n. 11, p.179-187, 1989.

MEYER, J. P; ALLEN N. J. Commitment in the workplace: theory, research and application. Thousand Oaks, CA: Sages Publications, 1997.

PAAUWEE, J; RICHARDSON, R. Introduction special issue on HRM and Performance. International Journal of Human Resource Management, v. 8, n. 3, p. 257-262, 1997.

ROTHWELL A; ARNOLD J. Self perceived employability, development and validation of a scale. Personnel Review, v. 36, n. 1, p. 23-41, 2007.

SILlA, I; DE CUYPER, N.; GRACIA, F.; PEIRÓ, J. y DE WITTE, H. Job insecurity and well-being: moderation by employability. Journal of Happiness Studies, v. 10, n.6, p. 739-751, 2009.

STUMPF, S; COLARELLI S; HARTMAN. Development of the career exploration survey. Journal of Vocational Behavior, v. 22, n. 2, p. 191-226, 1983.
SVERKE, M, HELLGREN, J; NASWALL, K. No security: a meta-analysis and review of job insecurity and its consequences. Journal of Occupational Health Psychology, v. 7, n.3, p. 242-264.

VAN DER HEIDJE; VAN DER HEIJDEN. A competent based and multidimensional operationalization and measurement of employability. Human Resources Management, v. 45, n. 3, p. 449-476, 2006. 